\title{
Percepción comparada de profesores y alumnos universitarios de la actividad docente y su incidencia en el rendimiento académico
}

\author{
María E. Martín-Palacio1, Cristina Di Giusto², Andrés Avilés-Dávila1, y Arquímedes Perlaza ${ }^{3}$ \\ (1) Universidad Complutense de Madrid, Facultad de Educación, Dpto. Investigación y Psicología en Educación. La \\ Almudena, Rector Royo Villanova, s/n, 28040 Madrid - España. (correo-e: mariaeugeniamartin@edu.ucm.es, \\ andresav@ucm.es) \\ (2) Universidad de Burgos, Dpto. Psicología Evolutiva y de la Educación. Calle Don Juan de Austria, s/n, 09001 Burgos - \\ España (correo-e: cristinadgv@gmail.com) \\ (3) Universidad Antonio Nariño, Dpto. Educación Cl. 58a Bis \#3794, Bogotá, Cundinamarca, Colombia \\ (correo-e: aperlaza51@hotmail.com)
}

Recibido Abr. 3, 2020; Aceptado Jun. 4, 2020; Versión final Jul. 30, 2020, Publicado Dic. 2020

\begin{abstract}
Resumen
Este trabajo analiza empíricamente la percepción que profesores y alumnos tienen de la actividad académica y su incidencia en el rendimiento. Se emplea el método instruccional de análisis de la situación educativa (MISE) con dos cuestionarios y en base a cinco principios: intencionalidad, diseño de instrucción, diseño de aprendizaje, relaciones interpersonales y sistema de evaluación. Participan 1.444 estudiantes y 68 profesores. Se realizan análisis de significación de diferencias y ecuaciones de regresión sobre el rendimiento académico. Los resultados indican que la percepción de la situación educativa presenta diferencias significativas en cuatro de los principios, y que no todos los principios influyen de igual manera sobre el rendimiento académico. No existen diferencias significativas entre profesores y alumnos en cuanto a la percepción de los procesos de aprendizaje. Se concluye que estos procesos junto con el proceso evaluador y la motivación o intencionalidad son los que tienen mayor efecto positivo en el rendimiento académico.
\end{abstract}

Palabras clave: aprendizaje; rendimiento; método instruccional; percepción; universidad

\section{Comparative perception of teaching activity between professors and university students and its impact on academic performance}

\begin{abstract}
This research study examines empirically the perception that professors and students have of academic activity and its impact on performance. The method used is the instructional educational situation analysis (IMESA) and was applied by using two questionnaires. It was based on five principles: intentionality, instruction design, learning design, interpersonal relationships, and evaluation system. The sample consists of 1,444 students and 68 professors. Data is analyzed by performing a significance analysis of differences and regression equations on academic performance. The results indicate that there are significant differences between the perceptions of four of the principles and that not all principles influence academic performance in the same manner. There are no significant differences between professors and students in perception of learning processes. It is concluded that these processes along with assessment processes and the motivation or intentionality have the greatest positive effect on academic performance.
\end{abstract}

Keywords: learning; performance; instructional method; perception; university 


\section{INTRODUCCIÓN}

A la pregunta formulada por Martín del Buey et al. (2010) de ¿Quién me ha robado la tiza? donde se plantea la invasión de la multimedia en el aula y el supuesto conflicto que ello puede acarrear, y a la apuesta por la educación mediática como un movimiento internacional imparable, Duart (2011) postula el gran reto de la Universidad que denomina hibridación. Entiende por ella, compaginar la docencia presencial tradicional con la existencia de otras plataformas virtuales donde es cada vez más asequible la adquisición del conocimiento y su forma de procesarlo más autónoma y autorregulada, aunque ello no implica la eliminación de la bondad de la presencialidad.

Es un hecho que la Universidad no puede estar al margen del tránsito de la generación Gutenberg a la generación Google (presente en los alumnos), como señalan Parodi et al. (2019). Son múltiples los trabajos centrados en el empleo por parte del profesorado y del alumnado de estos medios para la transmisión y adquisición del conocimiento. Respecto al profesorado universitario existe una preocupación tanto por conocer la frecuencia de empleo de las tecnologías, como por el tipo de actividades de aprendizaje utilizadas y la percepción y uso de las TIC. Respecto a los alumnos universitarios, también se ha analizado con crecido interés las bondades y las carencias que su empleo incide en su aprendizaje. Soler et al. (2018), muestran los cambios en la percepción de valor de los alumnos con la creación de contenidos a través de plataformas de información como, puede ser Wikipedia. No obstante, cabe señalar que el uso de estas tecnologías en modo alguno ha quitado entidad al papel mediador del profesorado ni ha desaparecido la necesidad de una relación interpersonal para que el proceso de aprendizaje se active. Esa interacción, es imprescindible.

Es una realidad verificada empíricamente que para el uso de estrategias de autorregulación del aprendizaje por el propio alumno y el desarrollo de estrategias de aprendizaje y habilidades de razonamiento se necesita potenciar la interacción social y la cooperación entre alumnos y profesores (Cerezo et al. ,2019). En este contexto se plantea concretar en qué términos debe definirse esa relación y medir el empleo que hace el profesorado de ella, así como el nivel de demanda de sus alumnos. Son altamente sugerentes los estudios presentados por lbarra y Benítez (2019) sobre la importancia del dialogo en el aula universitaria, los de Vargas-Díaz y Apablaza (2019) sobre la competencia comunicativa en la formación inicial del profesorado, el de Pérez-Villalobos et al. (2018), sobre de la habilidad de autocontrol del estudiante y la incidencia que tiene ello sobre el rendimiento académico y el relacionado con las competencias pedagógicas que caracterizan a un docente universitario realizado por Villarroel y Bruna (2017).

La concreción de esa relación presente en toda situación educativa debe reunir todas y cada una de las acciones que propician una acción dialogada entre profesores y alumnos. Estas no se limitan únicamente al momento de la exposición temática. Abarca otros momentos, previos y posteriores a ese acto que la didáctica general proclama también como de singular importancia. La preparación de la clase, la selección de los contenidos, su adaptación a las circunstancias sociales y personales de los receptores, la consideración de sus motivaciones y expectativas, la exploración de los conocimientos previos, de los usos y formas de aprendizaje utilizados, las preferencias por las distintas formas de evaluación utilizadas no dejan de ser concreciones de singular interés que forman parte dentro de una partitura de la comunicación.

El problema reside en conocer la importancia que en la actualidad tiene cada una de estas acciones en el rendimiento académico y el peso que tienen en él. Para ello es necesario definir mediante un exhaustivo y riguroso análisis todas aquellas variables o indicadores que están presentes en el aula. Ello constituye el objetivo de este trabajo. Se pretende conocer empíricamente su importancia en la acción docente universitaria y a esta pretensión se añade conocer qué peso tiene cada uno de ellos y su orden de prioridad sobre el rendimiento académico.

Para este menester se propone el Modelo Instruccional en Situación Educativa (MISE) formulado por Rivas (1997) y revisado por Doménech-Betoret $(2017,2018,2019)$ postulado en sus inicios sobre situaciones educativas basadas en la presencialidad en el aula, pero transferibles a situaciones semipresenciales y virtuales. Como más adelante describiremos en el MISE se plantea la existencia de cinco principios que están presentes en cualquier situación educativa. Estos principios son el de Intencionalidad, el Diseño Instruccional, el Diseño de Aprendizaje, el clima o las Relaciones Interpersonales en el aula y el sistema empleado de Evaluación.

Es obligado señalar, que la complejidad de la estructura y la multiplicidad de las variables presentes en toda situación educativa que el MISE describe, ha tenido como consecuencia que la riqueza que contiene no haya sido objeto de estudios más frecuentes desde sus planteamientos iniciales, observándose con todo que el número de investigadores va siendo cada vez mayor en España y en contextos latinoamericanos donde académicos como Francisco Martín del Buey (Universidad de Oviedo), Maㅡ Eugenia Martin Palacio 
(Universidad Complutense de Madrid) y Fernando Doménech-Betoret (Universidad de Castellón) han tenido especial empeño en su desarrollo.

Respecto al MISE han sido varias las tesis doctorales desarrolladas y defendidas, durante la primera y segunda decena del presente siglo dirigidas por los citados académicos. Estas investigaciones se han dirigido tanto a contextos de enseñanza media y profesional, como de forma más amplia a contextos universitarios donde bien se han considerado la totalidad de los principios del MISE o bien se han analizado de forma independiente cada uno de ellos.

\section{OTROS ANTECEDENTES}

EI MISE toma como referencia por una parte la Teoría de la Comunicación (Shannon y Weawer, 1972) cuyos elementos clave (Profesor / Currículum / Escolar) actúan siempre en triple interacción, y por otra parte la Teoría General de Sistemas (Bertalanfy, 1978) para explicar la regulación funcional de la información, entendiendo el proceso Enseñanza/Aprendizaje como un sistema semicerrado de comunicación. Como más adelante se señala, implica la presencia de cinco principios que interactúan en toda situación educativa.

EI MISE es un modelo sistémico, secuencial y jerárquico que parte de tres postulados teóricos que afectan a toda la estructura del modelo: Significación, Temporalidad Activa e Interacción. Este modelo, pretende convertirse en un modelo prescriptivo capaz de aportar datos para mejorar el proceso instruccional a través de cuestionarios que permiten evaluarlo, diferenciándose así de otros modelos descriptivos que han aparecido en las últimas décadas desde la Psicología de la Educación, que adolecen de la validación empírica para una situación educativa dada. EI MISE, parte inicialmente de un modelo teórico conceptual, para aportar posteriormente una vía metodológica del tratamiento de los datos obtenidos en la situación educativa, entendida ésta como el escenario real donde se produce la instrucción a través de la participación simultánea de los tres elementos claves en cualquier situación educativa presencial, semipresencial o virtual.

En este proceso interrelacional entre profesorado y alumnado el MISE considera cinco principios que deben estar presentes en cualquier situación educativa y que inciden de manera altamente significativa en el objetivo último de la educación: el desarrollo de las competencias académicas, personales y sociales del individuo. Estos principios son: Intencionalidad, Diseño de Instrucción, Diseño de Aprendizaje, Relaciones Interpersonales y Sistema de Evaluación. Cada uno de estos cinco principios se concreta en una serie de indicadores los cuales pueden ser evaluados a través de los cuestionarios MISE-R del profesor y alumno. Los resultados que se obtienen reflejan el nivel de interacción presencial, no presencial o virtual generado por el profesor y la percepción que se tiene de la misma por parte del alumnado. La presencia y activación de cada uno de estos cinco principios en cualquier situación educativa constituye una responsabilidad compartida en mayor o menor grado, según el principio, por parte de los alumnos y el profesorado y debe ser desarrollada de forma interactiva.

En el principio de Intencionalidad cuya finalidad básica es un cambio de estado del aprendiz hay tres momentos claves de cambio: el cognitivo, el personal y el de expectativas cuya responsabilidad compartida son del profesor y del estudiante. Por parte del profesor se concreta a nivel cognitivo en tener claro lo que los estudiantes deben aprender en la asignatura que imparten; en formular los objetivos en forma de competencias de forma clara y precisa y que éstos estén orientados a desarrollar diferentes niveles cognitivos y distintos dominios del estudiante (cognitivo, afectivo, social, etc.). El indicador personal se concreta en la realización de una evaluación inicial para conocer las características de los estudiantes (conocimientos previos, expectativas, intereses, etc.) y tener en cuenta la información obtenida para reorientar y ajustar el desarrollo de la asignatura. Finalmente, el indicador expectativas se concreta en explicar a los estudiantes de la utilidad e importancia de la asignatura para su formación y para su futuro profesional; en la transmisión de que su materia se puede superar sin problemas e incluso con buena nota, si se trabaja con interés; y en la observación de una buena predisposición hacia esta asignatura desde el inicio por parte de los estudiantes.

A nivel de alumnos el indicador "cambio de estado en el aprendiz", se concreta a nivel cognitivo en tener claro lo que debe aprender en la asignatura que se le imparte y si los objetivos están formulados de forma clara y precisa. A nivel personal en si se ha tratado de conocer conocimientos previos, expectativas, intereses, etc., y si esos conocimientos previos eran suficientes para afrontarla. $Y$ a nivel de expectativas en si el profesor le ha hecho ver la utilidad e importancia de esta asignatura para su formación y para su futuro profesional; si los objetivos son alcanzables; si hay esperanzas de obtener buenos resultados; y si hay motivación desde un principio para estudiar la asignatura. 
Respecto al principio del Diseño de Instrucción se señalan los siguientes indicadores: la estructuración de los contenidos, actividades y el control; estrategias de enseñanza; logística de recursos didácticos; temporalización y condiciones espaciales; y estrategias complementarias de individualización. Todo se traduce por parte del profesor en el aula en la necesidad de comunicar la selección de los conocimientos a tratar siguiendo criterios predefinidos tales como relevancia, utilidad e interés que ello tiene para sus alumnos; de comunicar con claridad las distintas modalidades de actividades (individuales, grupales, presenciales, a distancia, etc...) que deberán realizar para alcanzar los objetivos propuestos, con el peso que tiene cada una de ellas para la nota final; el tiempo estimado para su aprendizaje, los posibles itinerarios alternativos a elegir en base a la consideración de las posibles diferencias existentes entre los alumnos, y lugar y tiempo fijado para una atención individualizada.

Por parte de los alumnos, implica que estos perciben que la estructuración de contenidos y actividades y control, están, desde el principio, claramente expuestos y especificados en el programa. Asimismo, implica que se conoce el peso de cada actividad, el tiempo estimado para cada una y cómo se realiza la evaluación de las mismas. Que las estrategias de enseñanza son conocidas desde el principio junto con la metodología docente de la asignatura. Que la logística de recursos didácticos la conocen, así como los materiales necesarios para el desarrollo de la asignatura. Que las estrategias complementarias de individualización son conocidas por los estudiantes al igual que tiene conocimiento de las posibilidades de elección entre diferentes modalidades de aprendizaje y cómo puede hacer uso de las horas de atención tutorial del profesor.

Respecto al principio MISE relacionado con el Clima de Interacciones se presenta distintos niveles de interacciones en el aula: en el plano instruccional; en el plano socio-emocional; en las interacciones tutoriales y en las interacciones concretas en el desarrollo de los temas. El primer nivel establecido con las relaciones instruccionales los indicadores a nivel de profesorado se manifiestan cuando se facilitan los materiales necesarios para que los estudiantes puedan preparar adecuadamente los contenidos; se facilitan materiales complementarios para que los estudiantes puedan profundizar y ampliar; se realizan clases participativas y con un predomino del diálogo frente a clases expositivas; predomina el trabajo en grupo frente al individual; se estimula a que los estudiantes pregunten con total libertad cualquier duda surgida sobre los temas impartidos; se insiste en el aspecto práctico o aplicado de lo que se transmite; se utilizan materiales didácticos variados como elementos de apoyo; y se utiliza el aula virtual o se proponen actividades prácticas vinculadas con la teoría.

$Y$ a nivel de alumnado el nivel de relaciones instruccionales incluye aspectos como: poder disponer a tiempo de los materiales necesarios para preparar los temas; considerar si esos materiales han servido para facilitar el aprendizaje; disponer de materiales complementarios; disponer de clases dialogadas y participativas frente a las expositivas; utilizar trabajos grupales frente a individuales; estar estimulados para plantear cualquier duda; realizar actividades y ejercicios prácticos durante el desarrollo de los temas; facilitar la comprensión de los temas durante su explicación con diversos materiales; y disponer de un aula virtual operativa y aplicaciones prácticas.

El segundo nivel de relaciones en el plano socio-emocional se caracteriza por ser espontáneas y no estrictamente ligadas al proceso de enseñanza-aprendizaje, aunque constituyen una parte fundamental del clima de la clase. Las relaciones oscilan entre la colaboración y la competitividad y de los sentimientos del grupo al individualismo. En parte, estas relaciones también se encuentran suscitadas por las estrategias instruccionales que se utilizan en el proceso de enseñanza-aprendizaje. Los indicadores a nivel de profesorado son: si ha sabido mantener el control y el orden de la clase mientras impartía los temas; si ha favorecido y estimulado la participación; y si ha mostrado una disposición abierta y flexible para negociar con los estudiantes determinados aspectos como los plazos y las reclamaciones. A nivel de alumno, si se especifican los siguientes indicadores: si perciben preferencias o rechazos hacia algún estudiante; si se favorece y estimula su participación; y si se muestra disposición y flexibilidad para negociar y mantener relaciones fluidas y cordiales.

El tercer nivel referente a las interacciones tutoriales son relaciones de interacción mutua que representan esquemas de acción de difícil especificación. No obstante, incluye a nivel de profesorado: si se ha estimulado a los estudiantes a hacer uso de la atención del alumno tanto virtual como presencial; si se ha dedicado tiempo y esfuerzo atendiendo a los alumnos; y si la comunicación ha sido fácil y ágil. Se incluyen a nivel de alumnado si han percibido esos ánimos para el uso de la atención tanto virtual como presencial, si la comunicación ha sido ágil, fácil y si esta ha servido para potenciar el aprendizaje de la materia.

El cuarto y último nivel hace referencia a las interacciones a nivel concreto en el desarrollo de los temas. A modo de síntesis el profesor debe manifestarse como un facilitador de la comunicación propiciando la clase dialogada y participativa, la libertad de expresión y de pregunta, los trabajos grupales, la exclusión de 
prebendas y privilegios, el control del aula, la disposición abierta y flexible para negociar con los estudiantes y la cercanía en el trato personal e individualizado. Y así deben percibirlo de forma expresa y clara los alumnos.

El cuarto principio MISE está relacionado con el Proceso de Aprendizaje. Rivas (1997) señala los siguientes indicadores: i) El primer indicador son los conocimientos previos que hace referencia tanto a concepciones como a contenidos que se deben de tomar como nivel o punto de partida instrumental. En esta categoría se incluyen las concepciones erróneas personales que el estudiante aporta al inicio de la situación educativa. El profesor debe valorar la calidad de los conocimientos que tiene el estudiante para acomodarnos a ellos. ii) El segundo indicador es la adquisición de conocimiento que es el núcleo fundamental del proceso de enseñanza-aprendizaje que no se limita al proceso de asimilación o repetición de contenidos, sino que abarca también la interpretación, interiorización y construcción personal del aprendizaje. En este proceso el aprendiz es una persona activa, la protagonista de su aprendizaje. La responsabilidad de adquirir y construir conocimientos recae en el aprendiz, el cual despliega complejos procesos internos de los que sólo se tiene conocimiento una vez que se ha producido el aprendizaje. Pero el aprendizaje escolar también se ve favorecido y estimulado por acciones exteriores en la situación educativa. iii) El tercer indicador está relacionado con los procesos atencionales y de representación donde es necesario que el estudiante despliegue procesos para registrar, almacenar, interpretar u organizar la información, así como un sistema de representación que le permita dar una respuesta adecuada a las demandas del medio. Igualmente es necesario que el estudiante utilice claves precisas para seleccionar los aspectos fundamentales a estudiar y que mantenga esa acción de aprendizaje. iv) El cuarto indicador está relacionado con las estrategias, estilos y enfoques de aprendizaje. El modo en que un estudiante lleva a cabo las tareas del aprendizaje puede catalogarse como algo más que actuaciones azarosas ya que responden a modos de funcionamiento mental con cierta estabilidad, aunque modificables. v) El quinto y último indicador hace referencia a la temporalidad y transferencia. Rivas (1997) señala que la calidad del aprendizaje depende tanto del tiempo instruccional como del sentido personal, la transferencia e incitación a mantener la actividad o de la apertura a nuevas experiencias instruccionales.

La dimensión temporal está presente en todas las actividades de la situación educativa, teniendo una incidencia diferencial para los diferentes sujetos. El tiempo, sin duda es la dimensión donde se posibilita el Proceso de Enseñanza Aprendizaje el cual se puede separar en dos; "tiempos instruccionales" dedicados a la enseñanza y el "tiempo de aprendizajes". Este último va acorde a cada personalidad y no necesariamente a lo que nos indican los programas curriculares que se les entregan a los alumnos. Por otra parte, el tiempo dedicado a la enseñanza es fácilmente observable y medible, es regulado, planificado en base a las actividades y relaciones profesor alumno. Todos estos indicadores se analizan en el profesorado mediante los siguientes cuestionamientos: verificar mediante distintas técnicas de comunicación e interrogatorio si el seguimiento de las clases es satisfactorio, las dificultades son expresadas con libertad y facilitado su superación mediante las oportunas explicaciones, si hay interés por los temas y por la ampliación de los mismos, si se respeta los tiempos que el alumno necesita para tomar los apuntes y notas, y si el esfuerzo y tiempo que emplea en la adquisición de los conocimientos es suficiente. Y por parte del alumnado si esas acciones las percibe realizadas en sus profesores.

Finalmente respecto el ultimo principio del MISE hace referencia al sistema de control o Evaluación que tiene establecido el profesor donde es necesario mantener una comunicación constante con sus alumnos que favorezca la retroalimentación periódica con la intención de que ellos puedan mejorar la realización de tareas y trabajos, la información pronta de los errores cometidos, el fomento de la autoevaluación de los propios alumnos, la correspondencia de los ejercicios y actividades propuestas con los objetivos señalados en los temas y el comentario y análisis con los alumnos los resultados globales obtenidos y posibles pautas de mejora dialogadas. En base a lo expuesto se emplea el MISE en este trabajo pues contempla de forma integrada una serie de principios didácticos que están presentes en toda situación educativa, que guardan una estructura coherente, sistémica e interrelacionada.

Se considera que este abordaje tiene en si un carácter singular siendo una forma de abordarlo global y relacionado en todas sus partes. Se entiende que puede añadir valor a otras investigaciones de análisis más centradas en aspectos concretos o específico de una situación educativa dada. Entre ellos se encuentra los trabajos centrados en el Aprendizaje autorregulado, que son los más abundantes, aunque en lo relacionado con el rendimiento el número de ellos es menor (Hernández y Camargo, 2017). Las otras variables también han sido estudiadas de forma independiente tales como son el clima, la motivación, satisfacción (Jacobs et al. 2018) y la personalidad (Wach et al. 2016). En este trabajo se presentan, a través del MISE, diferentes variables en conjunto e interactuando en una situación educativa de una forma más sistemática, organizada e integral. Bajo ese prisma de análisis se formula el objetivo de este trabajo que consistente en valorar la percepción que profesores y alumnos universitarios tienen de la actividad académica desarrollada siguiendo la metodología MISE y su incidencia en el rendimiento académico. 
Las hipótesis del trabajo son: i) Las valoraciones que dan los alumnos y profesores respecto a la actividad docente desarrollada en la universidad presentan fuertes carencias en la totalidad de los principios analizados por el MISE. ii) La autovaloración del profesorado significativamente superior a la de los alumnos en todos los principios. iii) Existen diferencias significativas a la hora de valorar los profesores y alumnos su actividad docente. iv) La incidencia de estos principios respecto al rendimiento académico presentan el siguiente orden de importancia siendo la intencionalidad o motivación la primera condicionante o en su caso determinante del rendimiento académico, seguida de los procesos de aprendizaje empleado por los alumnos, el sistema de evaluación, el proceso de instrucción desarrollado por sus profesores y el clima escolar favorable experimentado en el aula.

Las valoraciones que dan los alumnos y profesores respecto a la actividad docente desarrollada en la universidad presentan fuertes carencias en la totalidad de los principios analizados por el MISE. Se plantea esta hipótesis en base a la supuesta crisis del sistema docente universitario donde son frecuentes las críticas respecto a la motivación por parte del profesorado hacia la actividad docente frente a su actividad investigadora que es más valorada y premiada a efectos de promoción profesional (Palali et al., 2018 y Cao et al., 2020). A ello se añade, la débil preparación didáctica que sé solicita con frecuencia para el acceso al cuerpo docente universitario donde se prioriza en el currículo de forma muy extensa la actividad y experiencia investigadora junto a un alto expediente académico, y a medida que se alcanzan estos niveles se recompensa con pérdida de la actividad docente considerada de menor rango y encomendada a profesores de menor nivel curricular. En España al menos este comportamiento suele ser muy habitual. Igualmente se sustenta la hipótesis en la escasa importancia que parecer darse al clima en el aula en estos niveles, dado que el trabajo prioritariamente es de carácter individual y la relación profesorado y alumno es muy personal y las actividades grupales no suelen contar con el suficiente respaldo en el profesorado. El ambiente competitivo prima sobre el cooperativo. Finalmente, la hipótesis se apoya en el sistema de evaluación más frecuente que suele estar centrado en una adquisición de los conocimientos dadas en el aula y una evaluación basada en el grado de conformidad con los mismos adquiridos por el alumno y repetidos miméticamente en el examen sin que se centre el tema en el proceso de aprendizaje utilizada para ello, ni se valore como tal.

La autovaloración del profesorado significativamente superior a la de los alumnos en todos los principios. Se plantea esta hipótesis bajo el supuesto bajo nivel de autocrítica del profesorado frente a su desempeño académico y el mayor desarrollo crítico y de disconformidad del alumnado sobre el modo operativo de proceder de sus profesores.

Existen diferencias significativas a la hora de valorar los profesores y alumnos su actividad docente. Los profesores valoran de mayor a menor su implicación en los cinco principios MISE: Diseño de Instrucción, Sistema de evaluación, Intencionalidad, Procesos de aprendizaje y Clima en el Aula. Los alumnos valoran de mayor a menor la percepción que tienen de sus profesores en su desempeño docente: Diseño de Instrucción, Intencionalidad, Clima en el aula, Procesos de aprendizaje y Sistema de evaluación. Se plantea esta hipotesis de trabajo bajo dos supuestos: i) El supuesto de que la autopercepción del profesorado sobre su desempeño docente valora de forma prioritaria su diseño de instrucción y su sistema de evaluación donde se considera que su nivel de responsabilidad o participación es mayor y con menor valoración la intencionalidad de los alumnos, los procesos de aprendizaje y el clima en el aula donde consideran que su nivel de responsabilidad es menor. ii) El supuesto de que los alumnos al ser receptores del desempeño docente de sus profesores no van a dar esos mismos índices de valoración, siendo la intencionalidad, los procesos de aprendizaje y el clima de relaciones interpersonales existentes en su situación educativa los más valorados y los peor valorados los procesos de instrucción empleados por sus profesores y el sistema de evaluación. Y ello parte del nivel de responsabilidad que se otorga en cada uno de los principios, considerándose la intencionalidad, el aprendizaje y el clima más responsabilidad de los alumnos y el sistema de instrucción y de evaluación con más carga de responsabilidad del profesorado.

La incidencia de estos principios respecto al rendimiento académico presentan el siguiente orden de importancia siendo la intencionalidad o motivación la primera condicionante o en su caso determinante del rendimiento académico, seguida de los procesos de aprendizaje empleado por los alumnos, el sistema de evaluación, el proceso de instrucción desarrollado por sus profesores y el clima escolar favorable experimentado en el aula. Se plantea esta última hipótesis en base al supuesto que la motivación o intencionalidad del alumno constituye la base que genera un buen rendimiento al igual que es la base que determina o condiciona cualquier actividad humana. Le sigue la forma o el diseño de aprendizaje 0 estrategias empleadas para su consecución en base a la importancia que cada vez se da al aprendizaje autorregulado por el propio alumno. En tercer lugar, el resultado académico se entiende que mantiene una estrecha dependencia con el sistema de evaluación empleado por el profesorado. En cuarto lugar, se concede menor incidencia al sistema de instrucción o diseño de instrucción empleado por sus profesores entendiendo que a los efectos finales el alumno suele permanecer en muchas ocasiones de una forma 
pasiva en ese proceso. Finalmente se parte del supuesto que el clima de las relaciones interpersonales que se dan en el aula tiene únicamente un efecto afectivo, pero no académico.

\section{METODOLOGÍA}

Para la consecución de los objetivos y la confirmación de las hipótesis formuladas se ha empleado una metodología de carácter descriptivo y empírico. Los instrumentos para llevar a término este trabajo han sido el MISE-R (Doménech, 2017) adaptada a población latinoamericana en versión diferenciada para alumnos y profesores. Son escalas tipos Likert cuyas puntaciones oscilan entre 1 minina y 5 máxima. El número total de ítems de la escala de alumnos es de 89 y el de profesores 94. Valoran por separado cada uno de los principios del MISE y el sumatorio de los mismos. En la descripción que se ha realizado de los cinco principios se deduce el sentido que tienen los ítems de que constan ambos cuestionarios. Se han analizados los índices de fiabilidad y validez confirmatorio de los supuestos estructurales que los conforman. En la tabla 1 se presentan los índices de fiabilidad (Cronbach, 1951) de los cuestionarios. En la misma tabla se muestran en la columna $n$ el número de ítems que corresponden a cada uno de los principios de la escala y la totalidad de ellos.

Tabla 1: Fiabilidades de los cuestionarios MISE. Alumnos y profesores

\begin{tabular}{|c|c|c|c|c|}
\hline & \multicolumn{2}{|l|}{ Estudiantes } & \multicolumn{2}{|l|}{ Profesores } \\
\hline & Alfa de Cronbach & $n$ & Alfa de Cronbach & $n$ \\
\hline Situación Educativa & .966 & 89 & .952 & 94 \\
\hline Intencionalidad & .836 & 12 & .732 & 13 \\
\hline Diseño de instrucción & .857 & 13 & .860 & 20 \\
\hline Clima de aula & .910 & 28 & .837 & 28 \\
\hline Procesos de aprendizaje & .914 & 23 & .915 & 19 \\
\hline Evaluación & .889 & 13 & .855 & 14 \\
\hline
\end{tabular}

Los índices de fiabilidad de ambas escalas superan en su totalidad los mínimos de aceptabilidad. En la tabla 2 se presentan los índices de ajuste al Modelo MISE corregido del cuestionario Alumnos.

Tabla 2: Índices de ajuste al modelo MISE corregido. Alumnos

\begin{tabular}{|l|c|c|c|c|}
\hline Índice de ajuste & Malo & Bueno & Modelo MISE & Modelo MISE corregido \\
\hline $\mathrm{X}$ 2ML/gl. & $>3$ & $\leq 2$ & 27.903 & 2.664 \\
\hline $\mathrm{p}$ & $<.01$ & $\geq .05$ & .000 & .046 \\
\hline RMSEA & $>.099$ & $\leq .05$ & .194 & .048 \\
\hline SRMR & $>.099$ & $\leq .05$ & .0473 & .0130 \\
\hline GFI & $<.85$ & $\geq .95$ & .934 & .995 \\
\hline AGFI & $<.80$ & $\geq .90$ & .803 & .977 \\
\hline $\mathrm{NFI}$ & $<.80$ & $\geq .90$ & .925 & .996 \\
\hline CFI & $<.85$ & $\geq .95$ & .927 & .997 \\
\hline
\end{tabular}

Todos los índices muestran un buen ajuste entre los datos al modelo a excepción de X2ML/gl, que muestra un ajuste moderado con relación al MISE de Alumnos. En la tabla 3 se presentan los índices de ajuste al modelo Mise corregido del profesorado.

Tabla 3: Índices de ajuste al modelo MISE corregido. Profesores

\begin{tabular}{|l|c|c|c|c|}
\hline Índice de ajuste & Malo & Bueno & Modelo MISE & Modelo MISE corregido \\
\hline $\mathrm{X}$ 2ML/gl. & $>3$ & $\leq 2$ & 4.133 & 1.255 \\
\hline $\mathrm{p}$ & $<.01$ & $\geq .05$ & .001 & .285 \\
\hline RMSEA & $>.099$ & $\leq .05$ & .361 & .103 \\
\hline SRMR & $>.099$ & $\leq .05$ & .0654 & .0383 \\
\hline GFI & $<.85$ & $\geq .95$ & .775 & .927 \\
\hline AGFI & $<.80$ & $\geq .90$ & .324 & .728 \\
\hline NFI & $<.80$ & $\geq .90$ & .834 & .960 \\
\hline CFI & $<.85$ & $\geq .95$ & .863 & .991 \\
\hline
\end{tabular}

Respecto al MISE profesores todos los índices muestran un buen ajuste entre los datos al modelo a excepción de GFI que muestra un ajuste moderado y RMSEA y AGFI que indican que los datos no se ajustan. A pesar de ello en su conjunto se considera un ajuste aceptable de los datos al modelo. 


\section{Muestra}

Los participantes del estudio se seleccionaron mediante un muestreo no probabilístico de carácter incidental. Se contó con 1444 estudiantes universitarios y 63 profesores universitarios. Los estudiantes eran originarios de dos países latinoamericanos, 719 (49.8\%) chilenos y $725(50.2 \%)$ colombianos, con edades comprendidas entre los 16 y los 24 años con una media de edad de 21.55 y una desviación típica de 3.204. La distribución por género fue de $587(40.7 \%)$ hombres y $851(58.9 \%)$ mujeres. Los 68 profesores del estudio fueron 25 (39.7\%) chilenos y $38(60.3 \%)$ colombianos. La distribución por género fue de $22(34.9 \%)$ hombres y $30(47.6 \%)$ mujeres. Los alumnos y profesores participantes cursaban distintas carreras universitarias de diferentes áreas: nutrición y dietética, terapia ocupacional, kinesiología y pedagogías en biología y ciencias, en educación física, en artes plásticas, en educación musical, tecnología, educación diferencial, educación parvulario, educación básica, química y ciencias sociales, castellano, filosofía, historia y geografía e inglés.

\section{Procedimiento de recogida y análisis de datos}

La recogida de datos de los cuestionarios se realizó en el último mes (diciembre) del curso académico 2018 una vez que las asignaturas objeto de estudio han sido impartidas en su totalidad y antes de los exámenes finales. Se aplicaron a todos los alumnos y alumnas voluntarios que han cursado la asignatura. El grado de participación, pese al carácter voluntario, fue mayoritario. Para evaluar el rendimiento académico las universidades proporcionaron las actas finales de las asignaturas objeto de estudio, previa autorización escrita y firmada de todos los participantes. La escala de valoración del rendimiento oscila entre el 1.0 a 7.0. La aplicación fue realizada por personal previamente entrenado y en presencia del coordinador del grupo de investigación del proyecto. Se aplicó en una sola sesión, en horario lectivo, en el aula de clase. El análisis de los datos se ha realizado utilizado los programas estadísticos SPSS 21.0 y AMOS 21.0. Para el análisis de la percepción de la situación educativa en el profesorado se han obtenido los estadísticos descriptivos de los profesores en el cuestionario tanto globalmente como agrupados por factores. Se ha seguido el mismo procedimiento con los 1444 alumnos.

Para determinar si existen diferencias en la situación educativa entre docentes y estudiantes se ha trabajado con las puntuaciones globales y las de los cinco principios. Dado que las observaciones recogidas por parte del profesorado son inferiores a 30 y existe una gran diferencia con el gran número de evaluaciones recogidas en el alumnado, se ha procedido a realizar la prueba no paramétrica $U$ de Mann Whitney. Para analizar la significatividad de las diferencias se ha procedido previamente al cálculo de $Z$ de Kolmogórov-Smirnov. Para el medir la significación de las diferencias de medias entre alumnos y profesores en el resto de los principios se ha procedido al cálculo de los estadísticos de contraste $U$ de Mann-Whitney y W. de Wilcoxon. Para determinar la relación entre la percepción de la comunicación y el rendimiento académico con relación a los principios de Intencionalidad, Diseño de Instrucción, Interacciones Personales, Diseño de Aprendizaje y Evaluación se ha llevado a término el análisis de regresión lineal.

\section{RESULTADOS Y DISCUSIÓN}

En la tabla 4 se presentan agrupados los descriptivos obtenidos en ambas muestras a los que se añade el error típico de la media.

Tabla 4: Descriptivos de ambas muestras

\begin{tabular}{|l|l|l|l|l|l|}
\hline & & Media & Desv. típ. & Rango promedio & Error tip. De la media \\
\hline \multirow{3}{*}{ Situación educativa } & Alumno & 3.94 & .50 & 78.88 & .01 \\
\cline { 2 - 7 } & Profesor & 4.24 & .38 & 84.89 & .04 \\
\hline \multirow{3}{*}{ Intencionalidad } & Alumno & 4.02 & .54 & 80.65 & .01 \\
\cline { 2 - 7 } & Profesor & 4.35 & .42 & 87.13 & .05 \\
\hline \multirow{3}{*}{ Interacciones personales Instrucción } & Alumno & 4.02 & .59 & 80.43 & .01 \\
\cline { 2 - 6 } & Profesor & 4.36 & .45 & 87.24 & .05 \\
\cline { 2 - 6 } & Alumno & 4.02 & .57 & 80.42 & .01 \\
\hline \multirow{2}{*}{ Diseño de Aprendizaje } & Profesor & 4.29 & .41 & 85.82 & .05 \\
\cline { 2 - 6 } & Alumno & 3.86 & .57 & 77.20 & .01 \\
\hline \multirow{2}{*}{ Evaluación } & Profesor & 3.94 & .55 & 78.88 & .06 \\
\cline { 2 - 6 } & Alumno & 3.76 & .73 & 66.84 & .01 \\
\cline { 2 - 6 } & Profesor & 4.28 & .48 & 85.78 & .06 \\
\hline
\end{tabular}


Las medias obtenidas presentan rasgos promedios altos tanto en alumnos como en profesores. Por proceder de una escala tipo Likert la puntuación mínima es 1 y la máxima posible 5 . Respecto a los alumnos la valoración más alta se centra por igual en los principios de intencionalidad, diseño de instrucción e interacciones personales. Las puntuaciones más bajas se dan en el principio de evaluación y en la puntuación total de la situación educativa. Por parte del profesorado la puntuación más alta se da en el principio de instrucción seguida en orden descendente por la intencionalidad, interacciones personales, evaluación, total de la situación educativa y diseño de aprendizaje. Las apreciaciones siempre son en todos los casos superiores la de los profesores. Para analizar la significatividad de las diferencias se ha procedido previamente al cálculo de Z de Kolmogórov-Smirnov (Ver tabla 5).

Tabla 5: Prueba de Z de Kolmogórov-Smirnov

\begin{tabular}{|l|l|l|l|l|l|l|l|}
\hline \multicolumn{2}{|l|}{} & $\begin{array}{l}\text { Situación } \\
\text { educativa }\end{array}$ & Intencionalidad & $\begin{array}{l}\text { Diseño } \\
\text { Instrucción }\end{array}$ & $\begin{array}{l}\text { Clima } \\
\text { Aula }\end{array}$ & $\begin{array}{l}\text { Proceso } \\
\text { Aprendizaje }\end{array}$ & Evaluación \\
\hline $\mathrm{N}$ & 1507 & 1507 & 1507 & 1507 & 1507 & 1507 \\
\hline $\begin{array}{l}\text { Parámetros } \\
\text { normales(a.b) }\end{array}$ & Media & 3.95 & 4.04 & 4.03 & 4.03 & 3.86 & 3.78 \\
\hline & Desviación típica & .50 & .54 & .59 & .56 & .57 & .72 \\
\hline $\begin{array}{l}\text { Diferencias } \\
\text { más extremas }\end{array}$ & Absoluta & .037 & .084 & .078 & .063 & .032 & .060 \\
\hline & Positiva & .026 & .040 & .051 & .047 & .024 & .049 \\
\hline & Negativa & -.037 & -.084 & -.078 & -.063 & -.032 & -.060 \\
\hline Z de Kolmogórov-Smirnov & 1.43 & 3.24 & 3.02 & 2.44 & 1.23 & 2.32 \\
\hline Sig. asintót. (bilateral) & .032 & .000 & .000 & .000 & .096 & .000 \\
\hline
\end{tabular}

Los datos obtenidos indican que las diferencias encontradas entre los profesores y alumnos en relación con su percepción de la situación educativa general y en detalle en cada uno de los principios objeto de análisis son significativas salvo en el proceso de aprendizaje. Dado que en Proceso de aprendizaje la significación a sintónica > (bilateral) es superior al 0.05 se procede al cálculo de Levene (Ver tabla 6).

Tabla 6: Prueba de Levene para el cálculo de significación de diferencias entre alumnos y profesores en Procesos de aprendizaje

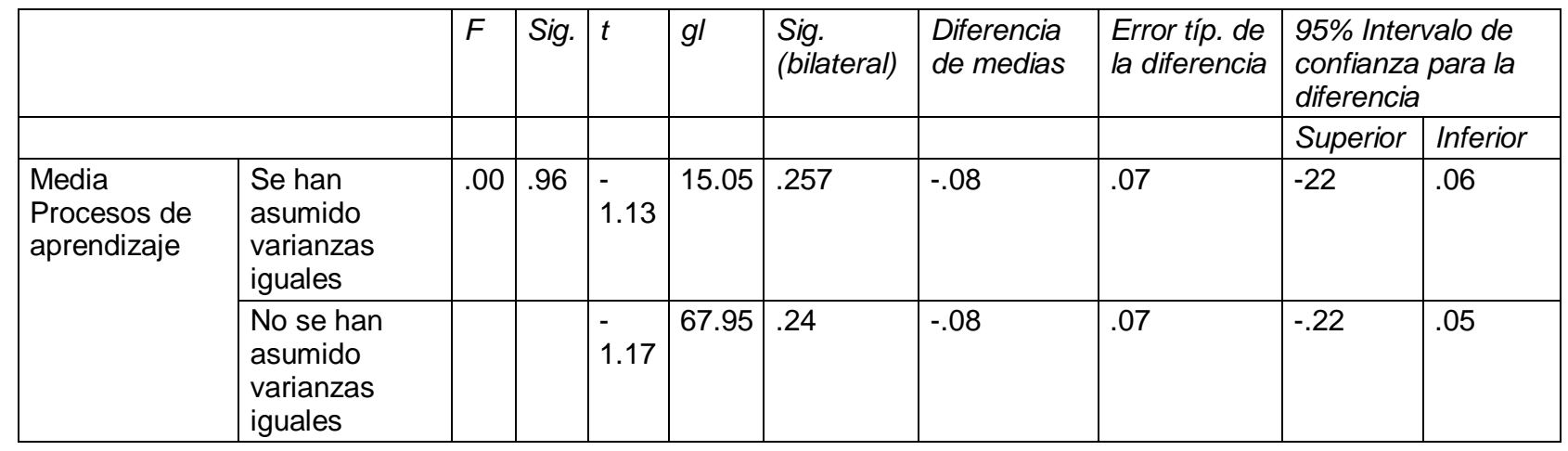

Se aprecia que la diferencia entre profesores y alumnos no es significativa en la valoración del proceso de aprendizaje. Para el medir la significación de las diferencias de medias entre alumnos y profesores en el resto de los principios se ha procedido al cálculo de los estadísticos de contraste $U$ de Mann-Whitney y W. de Wilcoxon donde se aprecia la existencia de diferencias significativas entre ambas medias. (Ver tabla 7).

Tabla 7: Estadísticos de Contraste

\begin{tabular}{|l|l|l|l|l|}
\hline & U de Mann-Whitney & W de Wilcoxon & Z & Sig. asintót. (bilateral) \\
\hline Situación educativa & 29302.000 & 25891.500 & -4.787 & .000 \\
\hline Intencionalidad & 29302.000 & 1073283.000 & -4.587 & .000 \\
\hline Diseño Instrucción & 29209.000 & 1072499.000 & -4.818 & .000 \\
\hline Interacciones personales & 32960.000 & 1072499.000 & -4.818 & .000 \\
\hline Evaluación & 25891.500 & 1069181.500 & -5.798 & .000 \\
\hline
\end{tabular}


Para determinar la relación entre la percepción de la comunicación y el rendimiento académico en relación con los principios de Intencionalidad, Diseño de instrucción, Interacciones personales, Diseño de aprendizaje y Evaluación se ha llevado a término el análisis de regresión lineal. Se pretende conocer la validez predictiva de los cinco factores del cuestionario MISE tomando como variable dependiente el rendimiento por parte del alumnado. La valoración global por parte del alumnado de su rendimiento resulta una media de 5.512 y una desviación típica de .8744. La puntuación máxima es de siete en las asignaturas. En la tabla 8 se muestra sus correlaciones con las puntuaciones en los distintos principios.

Tabla 8: Correlación de Pearson de los Factores del MISE con el Rendimiento Académico.

** La correlación es significativa al nivel 0.01 (bilateral).

\begin{tabular}{|l|c|c|}
\hline Escalas & Rendimiento académico & Sig. (bilateral) \\
\hline Intencionalidad & $.316\left(^{* *}\right)$ & .000 \\
\hline Diseño Instrucción & .045 & .120 \\
\hline Interacciones personales & $.180\left(^{(\star}\right)$ & .000 \\
\hline Diseño de Aprendizaje & $.425\left(^{* \star}\right)$ & .000 \\
\hline Evaluación & $.394^{(*)}$ & .000 \\
\hline
\end{tabular}

Excepto el factor Diseño de instrucción, todos los demás factores del cuestionario tienen correlaciones estadísticamente significativas con el rendimiento académico. El modelo planteado de cinco factores tiene un $R^{2}$ de .300 ( $\left.F=59.405, p=.000\right)$. Los coeficientes de regresión de esta variable se muestran en la tabla 9

Tabla 9. Valores de los estimadores de los coeficientes y significación estadística de la valoración del rendimiento académico.

\begin{tabular}{|c|c|c|c|c|c|}
\hline & \multicolumn{2}{|c|}{ Coeficientes no estandarizados } & \multirow{2}{*}{$\frac{\text { Coeficientes estandarizados }}{\text { Beta }}$} & \multirow[b]{2}{*}{$\mathrm{t}$} & \multirow[b]{2}{*}{ Sig. } \\
\hline & $\mathrm{B}$ & Error típ. & & & \\
\hline (Constante) & 3.300 & .263 & & 12.543 & .000 \\
\hline Intencionalidad & .031 & .007 & 205 & 4.696 & .000 \\
\hline Diseño Instrucción & -.032 & .006 & -.260 & -5.690 & .000 \\
\hline Interacciones personales & -.014 & .003 & -.235 & -4.350 & .000 \\
\hline Diseño de Aprendizaje & .029 & .003 & .409 & 8.195 & .000 \\
\hline Evaluación & .029 & .005 & .291 & 6.361 & .000 \\
\hline
\end{tabular}

La ecuación de regresión a través de los coeficientes estandarizados, que permita predecir, para cualquier sujeto de la población, su puntuación en el criterio conociendo sus puntuaciones en las variables predictoras, resultó la siguiente:

Rendimiento $\left(Y^{\prime}\right)=.205\left(X_{1}\right)+(-.260)\left(X_{2}\right)+(-.235)\left(X_{3}\right)+.409\left(X_{4}\right)+.291\left(X_{5}\right)$.

En esta ecuación: $Y^{\prime}$ es la puntuación pronosticada en expectativas de calificación, $X_{1}$ la puntuación del principio de Intencionalidad; $X_{2}$ la puntuación del principio Diseño de Instrucción"; $X_{3}$ la puntuación del principio Interacciones personales; $X_{4}$ la puntuación del principio Diseño de aprendizaje; y $X_{5}$ la puntuación del principio de Evaluación. El principio Diseño de aprendizaje es el que presenta mayor poder predictivo seguido en orden decreciente el sistema de evaluación, la intencionalidad y con carácter negativo el diseño de instrucción y las interacciones personales. A los efectos de dar cumplimiento al objetivo de valorar la percepción que profesores y alumnos universitarios tienen de la actividad académica desarrollada en las aulas siguiendo la metodología MISE y su incidencia en el rendimiento académico en este apartado se procede al análisis de los resultados y al cumplimiento o no de las hipótesis formuladas.

Respecto a la primera de la hipótesis planteada que vaticina que las valoraciones dadas por los alumnos y profesores respecto a la actividad docente desarrollada en la universidad presenta fuertes carencias y existen diferencias significativas a la hora de valorar la actividad docente, sorprende constatar las altas valoraciones, por encima de la media, en la totalidad de los principios MISE que tanto profesores como alumnos universitarios dan a la situación educativa presente en las aulas. En consecuencia, la hipótesis formulada no se cumple en su totalidad ni en ninguna de los principios que, si bien existen diferencias entre unos y otros, todos se valoran por encima de la media por parte del profesorado como del alumnado. Es significativo que sea el principio de instrucción uno de los más valorados.

La segunda de las hipótesis plantea que la autovaloración del profesorado significativamente superior a la de los alumnos en todos los principios. Los resultados obtenidos confirman la hipótesis de que el profesorado valora siempre la situación educativa con puntuaciones más altas en todos los principios MISE, 
y esas diferencias son significativas respecto a las expresadas por los alumnos salvo en las puntuaciones obtenidas en el principio relacionado con el Diseño de Aprendizaje cuyas diferencias no lo son. En consecuencia, se confirma la segunda hipótesis formulada. La explicación de estas diferencias en favor del profesorado proviene de la base de que el profesorado hace una autoevaluación de su quehacer docente, y normalmente en estas autoevaluaciones sobre su proceder se pierde algo de objetividad salvo que se tenga un alto sentido crítico de ello. Los alumnos en cambio la valoración que hacen es sobre la percepción que tienen del actuar docente de sus profesores, y en esos juicios es más frecuente una mayor discrepancia a lo que se añade mayor objetividad.

Cabe destacar, que tanto los profesores como los alumnos puntúen los desempeños en torno al principio relacionado con el Diseño de Aprendizaje como los de más baja puntuación y esas diferencias no sean significativas. Este resultado nos indica que en el Diseño de Aprendizaje es el que recibe mayor crítica o se percibe con mayor carencia. Esto parece afianzar la idea de la necesidad de enriquecer tanto por parte de los alumnos y profesores estos procesos en la medida que se reclama mayor insistencia en la autorregulación y autocontrol de los mismos por parte del alumnado y que el profesorado genere estrategias conducentes a ello. Más adelante se incide en este punto.

Respecto a la tercera hipótesis relacionada con las valoraciones dadas por los profesores y alumnos a la presencia de los cinco principios del MISE los resultados obtenidos en los profesores solo se confirman en cuanto a la valoración que dan de su Diseño de Instrucción, pero no en el orden de los principios, siendo la Intencionalidad, las Interacciones Personales, la Evaluación y Diseño de Aprendizaje frente al orden señalado en la hipótesis: Sistema de Evaluación, Intencionalidad, Procesos de Aprendizaje y Clima en el Aula.

Con respecto a los alumnos los resultados obtenidos confirman la hipótesis dado que la valoración más alta se centra por igual en los principios de Intencionalidad, procesos de instrucción e interacciones personales, las puntuaciones más bajas se dan en el principio de aprendizaje y en de evaluación. Centrando la atención en las valoraciones que presentan las puntuaciones más bajas tanto por parte del profesorado como por parte del alumnado, en el principio de Procesos de Aprendizaje se considera que estos datos debe ser objeto de una seria reflexión. Con respecto a esto último, es una realidad que los movimientos emergentes didácticos señalan la necesidad de desarrollar procesos de autocontrol y autorregulación del aprendizaje de las distintas materias. Los alumnos responden a esta necesidad solicitando un desarrollo más creciente de estos procesos en las aulas, pero señalan como significativamente carentes el uso que los profesores hacen del empleo de las estrategias que los desarrollan, los profesores por su parte refieren las frecuentes dificultades que encuentran para ello. Los resultados obtenidos en este estudio reflejan empíricamente esa realidad.

Respecto al Sistema de Evaluación los resultados aquí obtenidos están indicando que se impone la necesidad de fomentar una mayor interacción entre profesores y alumnos dado que aquellos aprecian que cumplen su cometido y los alumnos en cambio perciben que el proceso de evaluación seguido por sus profesores presenta significativas carencias. A raíz de estas consideraciones es oportuno señalar que en los resultados obtenidos se destaca la estrecha relación que se establece entre los Procesos de Aprendizaje (cuarto principio del MISE) y el Proceso de Evaluación (quinto principio del MISE) al igual que ocurre cuando se estudia el proceso del aprendizaje autorregulado, donde la autorregulación de la evaluación es un conjunto de soportes ofrecidos a diferentes niveles, en diferentes momentos y con diferentes propósitos (Rochera y Naranjo, 2007).

Álvarez (2009) contempla en los procesos de regulación los tres niveles secuenciados llamados: proactivo donde el docente puede acomodar la ayuda en la misma interacción que se produce como consecuencia de la actividad conjunta que realizan ambos alrededor de un contenido en el aula (regulación interactiva); interactivo donde el docente puede decidir ajustar algunos de los elementos del proceso de enseñanza y aprendizaje una vez acabada la sesión en el aula y después suponer la revisión de la planificación que se había previsto, o la inclusión de alguna actividad posterior; retroactivo donde el docente puede decidir volver sobre algunos contenidos trabajados a lo largo del proceso de enseñanza y aprendizaje una vez acabada la actividad de evaluación.

Esta regulación, con un carácter más retroactivo, supone el diseño de actividades de refuerzo después de la evaluación. En estos tres niveles se diluye para los teóricos del aprendizaje autorregulado de forma un tanto genérica los cinco principios MISE que los separa de una forma más minuciosa, detallada y diferenciada. De este modo, la evaluación puede constituirse en un instrumento fundamental para regular la intervención docente a lo largo del proceso de aprendizaje y a su vez en un elemento útil para que el alumno pueda autorregular su propio proceso de aprendizaje o en su caso la llamada retroalimentación (O’Donovan et al. 2016). 
La cuarta de la hipótesis relacionada con la incidencia de estos principios sobre el rendimiento académico no se confirma en su totalidad. El análisis de regresión refleja en primer lugar la importancia de los Procesos de Aprendizaje. Los resultados obtenidos reflejan empíricamente esa realidad: trabajar en el empleo y desarrollo de técnicas de aprendizaje va a determinar o en su caso condicionar una significativa influencia en el rendimiento académico.

El principio de Evaluación tiene el segundo peso más alto a la hora de determinar el rendimiento académico. De nuevo, los resultados relacionados con el proceso de evaluación están señalando dos aspectos de especial importancia. Por una parte, la necesaria activación e incremento de las pautas relacionadas con los procesos de Evaluación donde la participación del alumnado cobra especial importancia. Y en segundo lugar la relación estrecha entre los procesos de Aprendizaje y los de Evaluación, que en el análisis de la situación educativa propuesto por el MISE aparecen como principios diferenciados, pero estrechamente relacionados.

El principio de Intencionalidad ocupa el tercer lugar como peso en la ecuación de regresión. En relación con la intencionalidad o motivación presente en las aulas universitarias existen estudios que trabajan de forma independiente la influencia de esta variable, como los de Wentzel et al., (2010) o los estudios de Lin-Siegler et al., (2016). De forma más puntual sobre las implicaciones de las expectativas tenemos los estudios de Diniz et al., (2018), estos estudios se centran en las expectativas académicas de los estudiantes universitarios de primer año y en las diferencias de género. En los estudios mencionados se ha encontrado una relación entre la motivación y el rendimiento, pero están centrados más en la automotivación generada por el alumno que la propiciada por el profesorado.

En los resultados obtenidos en el MISE referentes a la Intencionalidad los datos son muy cercanos a lo óptimo y como tal son percibidos por los alumnos. A ello se añade que el nivel de influencia que tiene este dialogo intencional en relación con el rendimiento académico es positivo y forma junto con los Procesos de aprendizaje y el sistema de evaluación una relación directamente proporcional al mismo, pero en modo alguno es el principal determinante del rendimiento. Lo anterior, supone que una riqueza comunicativa en Intencionalidad, en los Procesos de Aprendizaje y en el Sistema de Evaluación inciden de forma positiva en el rendimiento. Pero todo ello en su justa medida, al menos en estos niveles universitarios donde se supone que ya el alumnado está más centrado en su interés y motivación por la materia objeto de estudio. Esta valoración contextualizada de la Intencionalidad o motivación en el aula en relación con los otros principios presentes en el MISE permite ajustar el valor que se debe conceder a ello. No obstante, no hay que dejar de considerar la importancia que tiene el nivel de expectativas de éxito que tanto el profesorado como el medio familiar tienen sobre el alumno como lo confirman los recientes estudios de Kremer et al. (2019).

Las relaciones interpersonales en las aulas universitarias analizadas de manera singular desde la perspectiva del clima escolar han sido relativamente frecuentes, siendo con todo más abundantes las centradas en niveles de secundaria y motivadas por la aparición de la violencia en estos centros. En relación con el rendimiento en contextos universitarios los resultados resultan contradictorios. Sandoval et al. (2017) no encontraron correlaciones entre las dimensiones de clima escolar con las calificaciones obtenidas por estudiantes, lo que significa que la percepción del entorno educativo no es un factor relacionado con el resultado de las evaluaciones en las asignaturas. Estos resultados, se contraponen a lo dicho por Bermúdez et al. (2015) cuando señalan que la apreciación del clima puede llegar a afectar el rendimiento académico de sus integrantes.

No obstante, la incidencia que tiene este principio de cara al rendimiento no tiene un carácter positivo y específico en base a los resultados obtenidos en el presente estudio, de cara al rendimiento. Ya se ha advertido que esas interacciones son favorecedoras de un clima positivo en el aula a modo de condición previo a un rendimiento, pero no tienen una repercusión directa sobre el mismo donde alcanza peso positivo la intencionalidad, el diseño de aprendizaje y el sistema de evaluación. En niveles universitario las interacciones propiciadas por el dialogo y la comunicación interpares y con el profesorado pierden en parte algo de la fuerza que tiene en otros niveles educativos. Sin quitarle su valor, la exigencia de un tipo de aprendizaje más centrada en el control y regulación personal genera que esas interacciones pasen, a efectos del rendimiento, a ocupar lugares más secundarios.

Finalmente, respecto al Diseño de Instrucción los datos indican que las actuaciones que se manifiestan en este principio por parte del profesorado y que son percibidas por el alumnado como claras, concretas, metodológicamente estructuradas y altamente valoradas tienen escaso o menor valor sobre el rendimiento académico. Este dato es revelador en la medida que indica que el proceso de instrucción basado en un Diseño de Instrucción muy programado y dirigido por el profesorado donde el alumno se hace de forma muy 
significativa en receptor o agente pasivo del mismo tiene baja o incluso negativa incidencia en el rendimiento. La ecuación de regresión indica que tiene un peso negativo.

Estos datos obtenidos están en línea con la idea de que la Educación Superior debe dar respuesta a una sociedad nueva, más práctica y globalizada donde los profesores deben concienciarse que está en proceso de cambio no solo estructural sino metodológico y en dicho cambio el alumnado se sitúa en el centro del proceso de Enseñanza / Aprendizaje. Ello implica que no existe una metodología ideal, sino que el uso de una u otra va a depender de la materia, del grupo de estudiantes e incluso de los propios espacios. No obstante las más recurridas por el profesorado y que entendemos por tanto le da mejor resultado en el proceso de enseñanza-aprendizaje son en primer lugar las prácticas a través de Tics, la exposición magistral, a la que los nuevos tiempos no ha conseguido dejar atrás, muy al contrario se ha visto reforzada al complementarla con otras técnicas como son el uso de medios audiovisuales o preguntas dirigidas y el role-playing, quizás por un intento de reproducir en nuestras aulas la realidad, a la que van a llegar el día de mañana nuestro alumnado.

En síntesis, una materia instruccionalmente muy programada y heterodirigida por el profesorado no genera por sí misma una influencia positiva en el rendimiento. Pero si lo genera la activación de unos procesos de aprendizaje y en un sistema de evaluación en donde la participación del alumnado sea mayor. Los datos obtenidos en torno al proceso instruccional en definitiva ponen de relieve la importancia que tiene centrar el proceso en las distintas formas que existen para la adquisición del conocimiento y medios de incorporarlos e interiorizarlos.

\section{CONCLUSIONES}

De acuerdo con los resultados de este estudio y de su discusión, de la comparación con resultados de otros autores y del detallado análisis presentado se pueden extraer las siguientes conclusiones:

1. La percepción de los cinco principios MISE de los alumnos y profesores presenta un nivel superior a la media, existiendo diferencias significativas, a favor del profesorado, salvo en lo relacionado con los Procesos de Aprendizaje donde no existen diferencias significativas.

2. Los alumnos perciben la situación educativa existente con puntuaciones altas en los principios de Intencionalidad, Procesos de Instrucción e Interacciones Personales seguidas de las otorgadas al principio de Aprendizaje y el de Evaluación.

3. La autopercepción del profesorado da la puntuación más alta en el principio de Instrucción seguida en orden descendente por la Intencionalidad, Interacciones Personales, Evaluación y Diseño de Aprendizaje.

4. Son los procesos de aprendizaje los de mayor efecto positivo en el rendimiento académico, seguido por el proceso Evaluador y la motivación o Intencionalidad.

5. Los procesos de Instrucción y las Relaciones Interpersonales seguidos en las aulas universitarias requieren una significativa reforma a los efectos de incidir de una forma más clara en el rendimiento académico.

\section{REFERENCIAS}

Álvarez, I.M., Assessment for Contributing to Self-regulated Learning, ISSN: 1696-2095, Electronic Journal of Research in Educational Psychology, 7(3), 1007-1030 (2009)

Bermúdez, J.J., Pedraza, A. y Rincón, C.I., El Clima Organizacional en Universidades de Bogotá desde la Perspectiva de los Estudiantes, ISSN 1607-4041, Revista Electrónica de Investigación Educativa, 17(3), 1-12 (2015)

Bertalanfy, L. V., Theorie Generale des Systemes. 30-53, Dunot, París, Francia (1978)

Cao, C., Shang, L., \& Meng, Q., Applying the Job Demands-Resources Model to Exploring Predictors of Innovative Teaching Among University Teachers. https://doi: 10.1016/j.tate.2019.103009, Teaching and Teacher Education. 89, 1$10(2020)$

Cerezo, R., Fernández, E., y otros 3 autores, Mediating Role of Self-efficacy and Usefulness Between Self-regulated Learning Strategy Knowledge and its Use, https://doi:10.1016/j.psicoe.2018.09.001, Revista Psicodidactica, 24(1), 1-8 (2019)

Cronbach, L.J., Coefficient Alpha and the Internal Structure of Test, https://doi:10.1007/bf02310555, Psychometrika, 16(3), 297-334 (1951)

Diniz, A. M., Alfonso, S., y otros 5 autores, Gender Differences in First-year College Students' Academic Expectations, https://doi:10.1080/03075079.2016.1196350, Studies in Higher Education, 43(4), 689-701 (2018) 
Doménech-Betoret, F., Un Modelo Instruccional para Guiar la Reflexión y la Investigación en el Aula: El Modelo de Calidad de Situación Educativa, https://doi:10.25115/ejrep.v11i29.1565, Electronic Journal of Research in Educational Psychology, 11(29), 239-260 (2017)

Doménech-Betoret, F., The Educational Situation Quality Model: Recent Advances. https://doi:10.3389/fpsyg.2018.00328, Frontiers in Psychology-Educational Psychology, 9, 1-20 (2018)

Doménech-Betoret, F., Gómez-Artiga, A. \& Abellán-Roselló, L., The Educational Situation Quality Model: A New Tool to Explain and Improve Academic Achievement and Course Satisfaction, https://doi:10.3389/fpsyg.2019.01692, Frontiers in Psychology-Educational Psychology, 10, 1-9 (2019)

Duart, J.M., The Net on Teaching Processes at the University, https://doi:10.3916/C37-2011-02-00, Comunicar, 19(37), 10-13 (2011)

Hernández, A. y Camargo, A., Autorregulación del Aprendizaje en la Educación Superior en Iberoamérica: Una Revisión Sistémica, https://doi:10.1016/j.rlp.2017.01.001, Revista Latinoamericana de Psicología, 49(2), 146-160 (2017)

Ibarra, S., V., y Benítez, A., A., El Diálogo en el Aula con Estudiantes de Nivel Superior en Clases de Humanidades para Ingeniería, https://doi:10.4067/S0718-50062019000100055, Formación Universitaria, 12(1), 55-64 (2019)

Jacobs, J., Girard, C. y Pudvan, L., Learners' Needs Satisfaction, Classroom Climate, and Situational Motivations: Evaluating Self-Determination Theory in an Engineering Context, https://doi:10.1109/FIE.2018.8658880, IEEE Frontiers in Education Conference 1-5 (2018)

Kremer, K., P., Huang, J., y otros dos autores, College Expectations of Eighth Grade Students: The Role of Learning Approaches and Parent Influences, https://doi:10.1016/j.childyouth.2019.104396, Children and Youth Services Review 104, 1-9 (2019)

Lin-Siegler, X., Dweck, C. S., and Cohen, G. L., Instructional Interventions that Motivate Classroom Learning. https://doi:10.1037/edu0000124, Journal of Educational Psychology, 108(3), 295-299 (2016)

Martín del Buey, F, A., Marcone, T.R., y Martin-Palacio, M.E., ¿Quién me ha Robado la Tiza? La Invasión de la Multimedia en el Aula. Del Conflicto a la Seducción sin Perder la Identidad, ISSN: 0214-9877, Internacional Journal of Developmental and Educational Psychology, 4, 689-696 (2010)

Palali, A., Elk, R., V., y otros dos autores, I. Are Good Researchers Also Good Teachers? The Relationship Between Research Quality and Teaching Quality, https://doi: 10.1016/j.econedurev.2018.03.011, Economics of Education Review, 64, 40-49 (2018)

Parodi, G., Moreno de León, T., y otros dos autores, Google or Gutenberg Generation: Chilean University Students' Reading Habits and Reading Purposes, https://doi:10.3916/C58-2019-08, Comunicar, 19(58), 85-94 (2019)

Pérez, V. M., Cobo, R. R., Sáez, F. M., y Díaz, M. A., Revisión Sistemática de la Habilidad de Autocontrol del Estudiante y su Rendimiento Académico en la Vida Universitaria, https://doi:10.4067/S0718-50062018000300049, Formación Universitaria, 11(3), 49-62 (2018)

O’Donovan, B., Rust, C. y Price, M., A Scholarly Approach to Solving the Feedback Dilemma in Practice, https://doi:10.1080/02602938.2015.1052774, Assessment \& Evaluation in Higher Education, 41(6), $938-949$ (2016)

Rivas, F., El Proceso de Enseñanza /Aprendizaje en la Situación Educativa, 83-102, Ariel, Barcelona, España (1997)

Rochera, M., J., y Naranjo, M., Fostering Self-regulated Learning in an Assessment Situation. ISSN: 1696-2095, Electronic Journal of Research in Educational Psychology, 13,5(3), 805-824 (2007)

Sandoval, M.C., Surdez, G., E., \& Samdoval, A.G., School Climate in the Engineering and Architecture Campus of a Mexican Public University: Students' Perspectives, https://doi:10.15359/ree.21-2.8, Educare. (21)2, 2-21 (2017)

Shannon, C., E., y Weaver, W., The Mathematical Theory of Communication, 117-128, University of Illinois Press, Illinois, US (1972)

Soler, A.J., Pavlovic, D. y Freixa, P., Wikipedia in Higher Education. Changes in Perceived Value Through Content Contribution, https://doi:10.3916/C54-2018-04, Comunicar, 54(26), 39-48 (2018)

Vargas-Díaz, C. y Apablaza, H., Competencia Comunicativa en la Formación Inicial Actual del Profesor de Matemática en Chile, https://doi:10.4067/S0718-50062019000300081, Formación Universitaria, 12(3), $81-90$ (2019)

Villarroel, V., A., y Bruna, D. V., Competencias Pedagógicas que Caracterizan a un Docente Universitario de Excelencia: Un Estudio de Caso que Incorpora la Perspectiva de Docentes y Estudiantes, https://doi:10.4067/S0718-50062017000400008, Formación. Universitaria, 10(4), 75-96 (2017)

Wach, F.S., Karbach J. y otros 3 autores, University Students' Satisfaction with their Academic Studies: Personality and Motivation Matter, https://doi:10.3389/fpsyg.2016.00055, Frontiers in Psychology, 7(55), 1-12, (2016)

Wentzel, K.R., Battle, A. y otros dos autores, Social Supports from Teachers and Peers as Predictors of Academic and Social Motivation, https://doi:10.1016/j.cedpsych.2010.03.002, Contemporary Educational Psychology, 35, $193-202$. (2010) 